\title{
Recomendaciones para la investigación psicológica con personas transgénero: Una aproximación desde una experiencia en Chile
}

\section{Recommendations for psychological research with transgender people: an approach from an experience in Chile}

Manuel Cárdenas Castro ${ }^{1}$, José L. Saiz Vidallet² ${ }^{2}$ Jaime Barrientos Delgado $^{3}$, Ricardo Espinoza-Tapia ${ }^{4}$, Dania Linker San Juan ${ }^{5}$, Fabiola Gómez Ojeda ${ }^{6}$, Mónica Guzmán González ${ }^{7}$ y Leonor Saavedra Lovera ${ }^{8}$

${ }^{1}$ Psicólogo. Doctor en Comportamiento Social y Organizacional. Investigador de la Facultad de Psicología de la Universidad de Talca. Investigador titular del Proyecto Anillos de Investigación en Ciencias Sociales y Humanidades, Universidad de Valparaíso.

E-mail: jose.cardenas@utalca.cl

${ }^{2}$ Psicólogo. Magíster en Psicología. Doctor en Psicología. Profesor titular del Departamento de Psicología de la Universidad de La Frontera, Temuco, Chile. E-mail: jose.saiz@ufrontera.cl

${ }^{3}$ Doctor en Psicología Social. Investigador asociado del Laboratorio Interdisciplinario de Subjetividad y Cambio Social (UAH-PUC) y en el proyecto Homosexualities: knowledge and methods del Institut d'Etudes Demographiques, Francia. Profesor en la Facultad de Psicología,

Universidad Alberto Hurtado, Chile. E-mail: jbarrientos@uahurtado.cl

${ }^{4}$ Psicólogo. Doctor en Psicología Social. Académico de la Escuela de Psicología de la Universidad Católica del Norte-Chile. Director del Observatorio de Infancias y Juventudes de la región de Antofagasta, Universidad Católica del Norte. E-mail: respinoza@ucn.cl ${ }^{5}$ Egresada de Trabajo Social de la Pontificia Universidad Católica de Chile, con Doble Major en Psicología y Trabajo social, y Minor en Trabajo social y familia. Coordinadora de investigación en Asociación Organizando Trans Diversidades Chile. E-mail: investigaciones@otdchile.org

${ }^{6}$ Psicóloga y Magíster en Psicología Social. Doctoranda en Psicología en la Pontificia Universidad Católica de Chile. E-mail: fagomez3@uc.cl

${ }^{7}$ Doctora en Psicología, diplomada en sexualidad humana, con formación de postítulo en terapia familiar y de pareja. Profesora titular de la Escuela de Psicología de la Universidad Católica del Norte. E-mail: moguzman@ucn.cl

${ }^{8}$ Socióloga. Miembro del equipo de investigación del proyecto Fondecyt 1170046 en la Universidad Diego Portales. E-mail: diego.saavedral@mail.udp.cl

Trabajo subsidiado por la Comisión Nacional de Investigación Científica y Tecnológica (CONICYT) de Chile mediante proyecto FONDECYT Nº1170046.

Facultad de Psicología, Universidad de Talca.

Región del Maule, Chile. 


\section{Resumen}

El presente trabajo expone y fundamenta recomendaciones basadas en la experiencia de investigación en personas transgénero, con la intención de que puedan ser útiles a quienes realicen estudios con dicha población. Estas recomendaciones incluyen orientaciones conceptuales, metodológicas y éticas, aplicables a lo largo del proceso de investigación. En particular, las orientaciones cubren los siguientes temas: definir con precisión el colectivo con el que se trabaja, registrar la variedad de identificaciones de género posibles al interior del mismo, utilizar métodos de muestreo aptos para poblaciones de difícil acceso, retribuir la participación, incluir líderes e investigadores transgéneros como expertos, pilotar los instrumentos en la población que será investigada, optar por modalidades de recolección grupal de datos, ofrecer asistencia individualizada durante la recolección, utilizar un lenguaje no estigmatizante e inclusivo, convocar la participación en conjunto con organizaciones transgénero, atender a la movilidad geográfica de la población, realizar una escucha comprometida y contemplar protocolos de derivación, considerar las diferentes trayectorias de transición, realizar sesiones de interanálisis y, finalmente, difundir apropiadamente los hallazgos al interior de la población transgénero. Se discuten estas proposiciones en términos de sus efectos en las comunidades trans, su utilidad y alcances éticos, su articulación con exigencias generales de toda investigación y sus implicaciones para el activismo.

Palabras clave: transgénero, orientaciones conceptuales, alternativas metodológicas, criterios éticos, implicaciones prácticas, Chile.

\section{Abstract}

Prejudice and discrimination have important effects on the well-being and quality of life of trans people. Hence, inves- tigating with said population implies, in addition to actions to obtain relevant and truthful information, having the necessary actions to attend to their needs and peculiarities. These are populations that are difficult to access (hard-to-reach), stigmatized, in which there is no defined sampling frame, that have physical and mental health indicators lower than those of any other group with which we have worked and that, therefore, it is necessary to take extreme the precautions not to reproduce types of relationships that may be harmful or offensive. The purpose of the present article was to systematize part of our field experience with transgender people, so that it can be useful for those who study with this population. These recommendations aim to provide some conceptual, methodological and ethical guidelines, useful throughout the research process. In particular, we made a series of recommendations that cover from the moment of taking conceptual decisions about the design of the study, through contact with the organizations, until the completion of the field work and subsequent stage of dissemination of results. In particular, a series of recommendations are made regarding: specifying the definition of the group with which one works, recording the variety of gender identifications as people present them, using to sampling methods for hard-to-reach populations or hidden populations, deliver a retribution for the participation (consider participants as experts to be compensated for their work), including activists as experts in every stage of the study (study design, field work, data analysis and dissemination of results), piloting the instruments in specific population, preferably opt for group applications modality and continue with a debriefing, have specific assistance for participants during the application (special needs derived from their age, educational level, reduced mobility, among others), use non-stigmatizing and inclusive language, call together with trans organizations (in order to establish greater trust in the population), attend to a mobility of the population (so as 
not to replicate the sample in different places), carry out a committed listening and contemplate referral protocols (if necessary given the type of situations described by the participants and their current degree of elaboration), consider different trajectories of transition, conduct inter-analysis sessions and disseminate appropriately within the transgender population. These propositions are discussed in terms of their effects on trans communities, their usefulness and ethical scope, their articulation with the general requirements of all research, and their implications for activism. On the other hand, these recommendations can serve as criteria for evaluating the ethical dimension of research projects with trans people. It may therefore be important to project reviewers, editors, and reviewers of manuscripts submitted for publication. The perspective that we assume in this work is aligned with those called "evidence-based activism", which implies articulating a variety of knowledge, scientifically accredited and of an "experiential" type, in order to explore situations in which the team members Research staff are part of networks that involve them personally in their study topics. It is precisely the relationship with groups that work for the rights of minorities, activists and members of these groups is essential, since they have knowledge acquired through their experience as part of discriminated groups and they can contribute to the mobilization of knowledge in the governance of health issues.

Keywords: Transgender, conceptual orientations, methodological alternatives, ethical criteria, practical implications, Chile

\section{Introducción}

La necesidad de realizar investigación en poblaciones vulnerables y de difícil acceso, como las personas transgénero (trans, de ahora en adelante), orientada éticamente para cautelar debidamente sus derechos (Adams, Pearce, Veale, Radix, Castro, Sarkar y Thom, 2017) y que resulte útil para las propias comu- nidades, constituye una exigencia insoslayable en la actualidad. Del mismo modo, cada una de las decisiones de investigación (el contacto inicial, el reclutamiento, la construcción y administración de instrumentos de medición, el análisis de los datos y la interpretación y la difusión de los hallazgos) requiere no solo resguardos éticos apropiados, sino también definiciones conceptuales y operacionales claras y estrategias metodológicas que permitan tener éxito en dichas tareas. No hacerlo implica que, para un estudio en marcha, los costos asociados, por ejemplo, a un lento proceso de reclutamiento, a la ausencia de respuestas fiables dada una escasa preocupación por un contacto inicial apropiado o la molestia derivada de una inadecuada difusión de los resultados al interior de la propia comunidad transgénero, puedan resultar enormes y difíciles de revertir.

Quizás una de las tareas más importantes es distinguir claramente el grupo que se quiere investigar. Hasta hace poco, los estudios incluían a las personas trans de forma poco afortunada, bajo el amplio concepto de población LGBTQ o LGBTI (lesbianas, gays, bisexuales, transgéneros, queer, intersexuales; Aspinall y Mitton, 2008; Keogh et al., 2006; Mitchell y Howarth, 2009), contribuyendo a confundir la orientación sexual con la identidad de género. No puede asumirse que las personas trans son individuos LGB, puesto que las primeras se definen por su identidad de género $\mathrm{y}$, en cambio, los segundos se definen por su orientación sexual (ver, más adelante, las definiciones de estas dos nociones). Además, a diferencia de estos últimos, las personas trans poseen un diverso rango de orientaciones. Del mismo modo, desde fines de la década de 1990, se ha cometido el error de agrupar indiferenciadamente a una serie de colectivos trans de características muy diversas (Whittle et al., 2007), tratándolos en la práctica como un grupo internamente homogéneo.

Las personas trans son aquellas que manifiestan una disconformidad entre su identidad 
de género y el sexo que se les ha asignado al nacer (APA, 2015). La existencia de personas trans en una amplia variedad culturas se encuentra debidamente documentada (Miller y Nichols, 2012) y, a pesar de su bajo reporte, se estima que ellas constituyen actualmente entre el 0.017 y $0.05 \%$ de la población general (Conron, Scott, Stowell y Landers, 2012; Meier y Labuski, 2013). En todo caso, es probable que esta población se encuentre subreportada, sea por las dificultades para recopilar información demográfica sobre las personas trans, o por tratarse de una población aún fuertemente estigmatizada y marginalizada en muchos países (Grant et al., 2011).

Si bien los estudios con población trans han sido más frecuentes en las últimas dos décadas (Bockting, Knudson y Goldberg, 2006; Coleman et al., 2012), persiste una importante brecha de conocimiento (McCarthy et al., 2015; Vincent, 2018). En Chile tenemos un claro rezago, ya que existen escasos estudios en población trans, y se han centrado principalmente en el prejuicio y la discriminación de que son objeto (Barrientos, Gómez y Cárdenas, 2016), sus experiencias de victimización (Barrientos y Cárdenas, 2014; Barrientos, Cárdenas y Saiz, 2016), el impacto que tal rechazo social genera en su bienestar psicológico (Barrientos, Cárdenas, Berendsen, Vidal y Meneses, 2013) y la subjetividad de los cambios asociados a sus procesos de tránsito (Casanova y Espinoza, 2018), también llamados de "afirmación de género" (y que refiere a las acciones emprendidas para desplazarse desde el sexo biológico a una expresión de género diferente). $\mathrm{Si}$ bien estos estudios han usado muestras pequeñas, ha sido posible realizar comparaciones con muestras de hombres gay, mujeres lesbianas y/o personas cisgénero (aquellas en que su identidad de género concuerda con su sexo biológico).

Las recomendaciones que aquí se presentan se elaboraron durante reuniones de coordinación y de interanálisis de datos y hallazgos y provienen de dos fuentes. Por un lado, desde los propios aciertos y errores en el aprendizaje de la actividad investigativa con esta población y por otro, desde la constatación de que no se recibe una adecuada capacitación sobre estos temas durante la formación universitaria, por lo que hubo que familiarizarse en la práctica misma de investigar. Esta insuficiente capacitación, que sigue siendo un problema común en Chile como en otros países (APA TFGIGV, 2009) puede ocasionar, como efecto no intencionado, consecuencias negativas en la población investigada, a la cual se desea, paradójicamente, favorecer (APA, 2015; Mikalson, Pardo y Green, 2012). Un ejemplo de ello es la fatiga en la población debido a su recurrente participación en un gran número de estudios, muchos de los cuales pueden tener fallas metodológicas o descuidos éticos (Harrison, Grant y Herman, 2012; Tagonist, 2009; Vincent, 2018).

Las recomendaciones aquí ofrecidas se limitan a la propia experiencia con población trans adulta, ya que entendemos que el trabajo con menores (niños, niñas y adolescentes) tiene una complejidad y especificidad propia. Es importante precisar que estas recomendaciones no pretenden agotar todas las posibilidades ni erigirse en una directriz para investigadores, sino que simplemente intentan transmitir esa experiencia para ponerla a su servicio, así como abrir un necesario debate respecto a las particularidades de la población trans a ser consideradas en el proceso de investigación.

\section{(1) Contar con una definición precisa del colectivo a estudiar}

Como se anunciaba en la introducción resulta equivocado incluir acríticamente a las personas trans dentro del colectivo general LGBTI, ya que este corresponde a un paraguas teórico que asimila de forma forzada y errónea las necesidades y características de un conjunto disímil de poblaciones, lo cual puede invisibilizar a los grupos que poseen una mayor vulnerabilidad (Staples, Bird, Masters y George, 2018). 
En el contexto chileno, en el que existe un marcado machismo y heterosexismo (Barrientos y Cárdenas, 2014; Cárdenas, Barrientos, Gómez y Frías-Navarro, 2012; Nierman, Thompson, Bryan y Mahaffey, 2007), la diferenciación percibida entre las varias minorías sexuales o de género es aún más acusada, lo cual produce a su vez amplias desigualdades sociales y culturales, así como elevados niveles de discriminación hacia las personas que trasgreden los mandatos de rol de género estrechamente vinculados al sexo asignado al nacer. De allí, la primera recomendación, relativa a una definición clara y precisa del colectivo con el cual se desea trabajar: las personas trans.

La distinción entre identidad de género y orientación sexual resulta fundamental. Cuando se habla de personas trans, se sitúa el tema en el campo de la identidad de género, esto es, la vivencia subjetiva del individuo respecto a su género, el cual puede o no coincidir con el sexo que le fue asignado al nacer. Como ya se señaló, en las personas gay, lesbianas o bisexuales el elemento definitorio es su orientación sexual, entendida como un patrón duradero de atracción afectiva y/o sexual hacia personas de su mismo sexo, de otro sexo o de ambos (Hereck, 2010). Por ello, las denominaciones "heterosexual", "homosexual" (lesbiana, gay) o "bisexual" refieren a las preferencias afectivo/sexuales de estas personas, con independencia de su identidad de género. La orientación sexual y la identidad de género son dimensiones diferentes que pueden solaparse o no. En las personas trans, el elemento definitorio es la falta de concordancia entre su sexo asignado al nacer y su vivencia subjetiva del género, y pueden tener ellas diferentes orientaciones sexuales.

Por otra parte, las personas trans deben ser diferenciadas de otros grupos que evitan definir su identidad de género en términos binarios (masculino o femenino) y/o sienten que su identidad varía o fluye continuamente a lo largo del tiempo. Asimismo, ellas deben ser diferenciadas de personas habitualmente nominadas bajo otros rótulos, tales como transexuales (quienes se han sometido a tratamientos de reasignación de sexo, incluyendo o no cirugía constructiva), travestis (quienes gustan de utilizar prendas de vestir asociadas al sexo opuesto) o intersex (individuos con genitales que tienen características biológicas de ambos sexos o cuyos genitales son ambiguos). Estas distinciones son importantes puesto que, por imposiciones culturales, se suele tratar a las personas trans de manera binaria, asumiendo que son categorías discretas directamente vinculadas con el sexo masculino o femenino asignado al nacer (Bockting, 2014), cuestión que podría reforzar la marginalización y la discriminación hacia las personas trans que no consienten dicho binarismo (Wolf y Dew, 2012). Estas diferenciaciones tienen repercusiones prácticas; por ejemplo, pueden orientar la construcción de un instrumento de medición o una pauta de entrevista, así como guiar el proceso de reclutamiento al constituirse ellas en criterios específicos de inclusión o exclusión de los participantes.

Si bien todos los colectivos agrupados bajo la categoría general de LGBTI comparten ciertos rasgos comunes, es importante reconocer las especificidades de cada colectivo y considerar que ellas derivan de su particular proceso de inserción social en un contexto específico que, muchas veces, involucra una mayor vulnerabilidad. Suelen abundar los estudios que incluyen a algunos o a todos estos grupos y analizan los datos sin desagregarlos, pudiendo con ello invisibilizar las particularidades de cada uno. Este es un aspecto muy relevante; se trata de poblaciones que requieren un tratamiento diferenciado pues, dependiendo de los contextos específicos, ellas poseerán capas adicionales de vulnerabilidad (Luna, 2008).

En este sentido, resulta también recomendable conocer la historia global y local de los grupos en los que se trabaja (Vincent, 2018). Investigar en colectivos trans requiere realizar una revisión acabada de su historia a fin de comprender sus peculiaridades y la situación 
de vulnerabilidad en que suelen encontrarse, como es el caso en Chile.

\section{(2) Registrar la variedad de identidades de género de los participantes}

Las personas trans suelen emplear una amplia diversidad de expresiones para describir su identidad de género (Meier y Labuski, 2013). La experiencia investigativa en Chile confirma una gran heterogeneidad de expresiones en el autorreporte de la identidad de género incluyendo algunas tales como: "hombre", "femenina", "mujer trans", "trans", "transexual", "queer", "homosexual”, “agénero", “mujer en cuerpo de hombre", "fluida/o", "persona (hombre y mujer) con expresión más masculina" y también expresiones peculiares como "chica transmarika", "divina" o "vampiro travesti".

Como la identidad de género es un elemento central en la investigación con población trans, el registro de esta identidad debe ser suficientemente sensible para captar la heterogeneidad con que ella es reportada por los participantes. Tal heterogeneidad puede deberse, en parte, a que la forma binaria de identidad ha significado durante mucho tiempo un ejercicio de dominio social sobre las personas trans al que muchas de ellas intentan rebelarse, sea oponiéndose a tales imposiciones normativas o buscando rutas de expresión más acordes con lo que ellas realmente sienten. Una primera estrategia para registrar la identidad de género es presentar una pregunta seguida de un conjunto de opciones de respuesta cerrada. Por ejemplo, Grant y colaboradores (2011) emplearon 15 diferentes opciones de identidad. Aunque el uso de esta estrategia facilita la codificación de la respuesta, impone al menos dos dificultades: se debe ofrecer un extenso listado de opciones $\mathrm{y}$, además, nunca habrá certeza de que ese listado es realmente comprehensivo de la diversidad de identidades que se pretende abarcar. Evaluando la experiencia de personas trans luego de contestar un cuestionario, Staples y colaboradores (2018) encontraron que, pese a ofrecer 14 opciones de identidad de género, algunos participantes señalaron que las opciones eran insuficientes. Si los investigadores decidieran emplear opciones de respuesta cerrada, sería altamente aconsejable agregar también una opción final de respuesta abierta que recoja identidades no incluidas en las opciones precedentes, lo cual permitiría una mayor sensibilidad a la hora de captar e incluir elementos identitarios relevantes para quienes participan de un estudio.

Una segunda estrategia para registrar la identidad de género, más recomendable que la anterior puesto que captura mejor las formas únicas de construcción subjetiva de la identidad, es emplear una pregunta con respuesta abierta, por ejemplo, “¿Cómo describirías tu identidad de género en tus propias palabras?" (Meier y Labuski, 2013; Krueger et al., 2020). Esta estrategia permite recoger la variedad de identidades de género de las personas trans, pero requiere, como tarea adicional, clasificar posteriormente las respuestas en categorías más inclusivas a fin de hacerlas susceptibles de análisis. Aunque esta clasificación podría ser construida por los investigadores a partir de las respuestas de los participantes, complementariamente se pueden usar clasificaciones ya elaboradas. Por ejemplo, la clasificación construida por Giami y Beaubatie (2014) incluye seis categorías de identidad de género: mujer, hombre, mujer trans, hombre trans, trans, y otras identidades. El empleo de clasificaciones previas sería ventajoso ya que evita la construcción de una clasificación propia, ofrece categorías operacionalmente definidas y ejemplos que facilitan la categorización, proporcionando confianza en que las categorías son suficientemente exhaustivas y permiten la comparación de resultados con otros estudios que hayan usado la misma clasificación. Esta práctica, sin embargo, no debe ser aplicada mecánicamente; antes, debe examinarse si la clasificación es pertinente en la población transgénero de interés $y$, si fuese necesario, modificarla. Así, por ejemplo, considerando las particularidades de los colectivos trans estudiados, la clasificación de Giami y 
Beaubatie, ya descrita, fue adaptada en Brasil (Carrara et al., 2019) omitiendo las categorías trans y otras identidades y agregando travesti y no binario. En Chile solo agregamos travesti y no binario a la clasificación de Giami y Beaubatie, generando así una taxonomía de ocho categorías.

\section{(3) Incluir estrategias de muestreo apropiadas para población transgénero}

El muestreo en poblaciones de difícil acceso siempre resulta complejo, sea porque no existen marcos apropiados a los cuales acudir para seleccionar la muestra o debido a que sus integrantes pertenecen a grupos socialmente estigmatizados, situación que los obliga a ocultarse o a encubrir su identidad. En Chile, las personas trans constituyen una población de difícil acceso, hecho agravado por ser un grupo estigmatizado y por la inexistencia de datos censales que permitan su estimación poblacional y la carencia, hasta hace poco, de derechos básicos que permitiesen su presentación pública con un nombre y sexo coherente con su identidad de género.

Varios procedimientos de muestreo son apropiados para este tipo de poblaciones, aunque para esta investigación ha sido particularmente útil la técnica denominada Respondent Driven Sampling (RDS; Heckathorn, 2002, 2007). El RDS corrige algunas de las debilidades metodológicas de los clásicos métodos de muestreo en cadena, como bola de nieve o informantes clave. Para ello, el RDS provee una estimación matemática de la representatividad de la muestra obtenida (a través de análisis de redes), sin desestimar que los propios miembros poblacionales son los más aptos para reclutar a otros miembros. Además, el RDS permite calcular estimadores insesgados y el error estándar de los intervalos de confianza.

El RDS comienza con el reclutamiento de un número limitado de semillas (o informantes iniciales seleccionados en virtud de la profundidad de sus vínculos sociométricos o de su particular posición en el entramado de relaciones sociales de la población de interés), número que se expande en progresivas olas de reclutamiento de pares. Las semillas reclutan solo en la primera ola, en tanto que los reclutados en ella toman la responsabilidad de traer nuevos participantes en una segunda ola $\mathrm{y}$ así sucesivamente. Cada participante recluta una cuota fija de personas, y recibe un doble incentivo: primero, se remunera a la persona cuando contesta un cuestionario o una entrevista y segundo, también se remunera una vez que las personas reclutadas por él asisten para completar el cuestionario o ser entrevistadas. La experiencia observada con diversas poblaciones indica que este procedimiento opera bastante bien, incluso cuando solo tenemos posibilidad de entregar un solo incentivo o ninguno (Barrientos, Cárdenas, Gómez y Guzmán, 2014; Cárdenas y Yañez, 2012).

La teoría subyacente al RDS asume que en un número suficientemente largo de olas de reclutamiento la composición de la muestra se estabiliza (Abdul-Quader, Heckathorn, Sabin y Saidel, 2006), hasta llegar a un estado en el cual los estimadores convergen y no cambian durante los subsecuentes ciclos de reclutamiento (se estima que luego de la quinta ola de reclutamiento la muestra debería alcanzar tal equilibrio). Este criterio de saturación, expresado por la estabilidad porcentual en categorías grupales significativas para el estudio (sexo, grupos de edad, etcétera), indicará cuándo detener el proceso de reclutamiento y permitirá, dado el reporte del tamaño de las redes sociales, estimar el tamaño de la población y calcular los intervalos de confianza de las estimaciones de tales parámetros poblacionales (Salganik, 2006). Estas estimaciones proveen el rango dentro del cual se espera encontrar el valor del parámetro, con algún nivel de confianza, por medio de la generación iterativa de muestras mediante un proceso de auto-reposición o bootstrap (Salganik, 2006; Salganik y Heckathorn, 2004).

Independientemente del método de muestreo elegido, es importante considerar que siempre se necesita el apoyo de las organiza- 
ciones y grupos más reconocidos en temas de la población trans ya que, como se verá más adelante, ellos otorgan validez a una investigación en la medida en que permiten garantizar el acceso a la población y la supervisión sobre los contenidos incluidos y los materiales utilizados (uso de lenguaje apropiado, definiciones claras, detección de subgrupos en la muestra, etcétera). En este sentido, resulta apropiado que personas de la comunidad trans o profesionales ligados a ella actúen como consultores en los estudios, y que sean debidamente reconocidos y visibilizados. El escenario ideal sería la incorporación de personas trans en los equipos de investigación a fin de que participen activamente en todas las fases investigativas, independientemente de que su oficio sea o no la investigación.

\section{(4) Entregar una retribución por la participación}

Si se utiliza el RDS, la retribución monetaria por participar es parte inherente a esa técnica muestral. Si se emplean otras técnicas (por ejemplo, bola de nieve), igualmente puede ser ventajoso incorporar algún tipo de retribución. Las personas trans habitualmente integran grupos vulnerables $\mathrm{y}$, muchas veces, con evidentes problemas económicos. Si bien hay variabilidad al interior de esta población, particularmente entre las personas más jóvenes, los datos disponibles en Chile indican que se trata de una minoría desfavorecida (Barrientos y Cárdenas, 2014), situación que es reflejada por los menores niveles de salud mental y bienestar psicológico, social y material que poseen respecto a la población cisgénero (Baner et al., 2009). En cualquier caso, se trata de considerar a los y las participantes como expertos (Hale, 2009) a los que se debe retribuir por su tiempo o, al menos, facilitar su participación mediante una compensación monetaria que implique que no tengan que incurrir en gastos adicionales (como gastos en transporte público) o reducir sus ingresos (como no trabajar durante el lapso que toma su participación), lo que muchas veces los alejan de la posibilidad de ser incluidos en un estudio. Si estas situaciones no son previstas, la posibilidad de inclusión en un estudio puede verse notoriamente disminuida, poniendo en riesgo la obtención de una muestra suficientemente variada.

Como ya se indicó, en el caso del RDS, la retribución puede entregarse no solo al colaborar como participante, sino también como reclutador de otras personas de su comunidad. Dicha retribución en ningún caso debe ser demasiado elevada a fin de no condicionar externamente la participación como sería el caso, por ejemplo, al colaborar solo por el incentivo o aceptar la invitación a participar cuando, en realidad, no se quiere participar. La retribución jamás debe constituirse en un elemento de presión para la participación ni impedir el abandono de un estudio del que no se desea seguir siendo parte. Así, la retribución debe ser entregada tanto a quienes completan su participación como a aquellos que la inician pero no la concluyen.

Según esta experiencia, las retribuciones son bienvenidas por la población trans, sobre todo ahora, cuando se invita cada vez más frecuentemente a sus miembros a participar en diversos estudios y por tanto, deben destinar tiempo y recursos para ello. Está bien establecido que el uso de estrategias de compensación permite mejorar las tasas de respuesta y captar mejor la diversidad de personas que componen este colectivo (Staples et al., 2018). La ausencia de retribución puede perjudicar a los miembros más desfavorecidos respecto a los miembros más acomodados de su propia comunidad; si los primeros se ven excluidos de participar por razones económicas, tal exclusión reproduce la desventaja inicial y además, incrementa su invisibilización.

La entrega de retribuciones es ciertamente un asunto controvertido, especialmente en poblaciones vulnerables, por cuanto se pretende contribuir a su bienestar y al mismo tiempo, mantener su autonomía. El empleo de retribuciones debe apuntar únicamente a 
compensar a la persona por los menoscabos o costes económicos que su participación en el estudio pueda haberle ocasionado. Por tal razón, estas compensaciones deben ser proporcionales a dicho detrimento y como ya se dijo, no excederlo de manera importante, evitando así subordinar la respuesta de la persona o inducirla a tomar una decisión que previsiblemente no tomaría en caso de no existir dicha retribución. La regla que se tuvo en cuenta en esta investigación es que se buscaba el consentimiento solo bajo circunstancias que brindaran al eventual participante la oportunidad de considerar libremente si colaboraba o no, procurando minimizar el riesgo de coacción sobre su decisión (Emanuel, 2005).

\section{(5) Incluir a personas trans en calidad de expertos}

La incorporación de personas trans como miembros de un panel de expertos provee un apoyo fundamental a los estudios con esta población. Tal iniciativa debe incluir no solo a investigadores trans, sino también a dirigentes y miembros de agrupaciones de protección de sus derechos (sindicatos, asociaciones, colectivos, etcétera) e integrantes de la propia comunidad. A estas personas se les debe reconocer su calidad de expertas, ya que poseen conocimientos y experiencias de los cuales, en principio, el resto carece y que pueden ser de utilidad en la investigación misma. Admitir estas carencias y reconocer los propios privilegios exigen un necesario sentido de humildad (Hale, 2009).

El panel de expertos/as podrá orientar, incluso desde la confección del proyecto de investigación, en aspectos como definiciones conceptuales y operacionales de variables relevantes, uso de un lenguaje apropiado, orientación para acceder a las diferentes muestras (no hay un solo tipo de trans ni un solo discurso respecto de lo trans, sino que existen muchas voces y muchas comunidades), así como sobre las posibles interpretaciones de los hallazgos. El panel también puede jugar un rol clave a la hora de diseminar los resultados y ponerlos al servicio de la comunidad.

La contribución individual de los miembros del panel debe ser reconocida formalmente, ya sea retribuyendo apropiadamente su participación, incluyéndolos en el equipo de investigación (esta es la opción óptima, aunque hay fuentes de financiamiento que no permiten incorporar personas sin las debidas credenciales académicas) o incorporándolos, según la naturaleza de su colaboración, como coautores en comunicaciones científicas. Más allá de la opción elegida, lo que resulta claro es la necesidad de considerar a las personas transgénero como verdaderas expertas y visibilizar sus aportes.

Este punto resulta fundamental, pues se trata de una opción ética por el reconocimiento de los/as otros/as integrantes de la comunidad trans con que se trabaja y un interés genuino por sus vidas, el cual se opone al denominado extractivismo académico (extraer información en las comunidades con fines investigativos sin generar, a cambio, ningún beneficio en ellas).

\section{(6) Realizar el diseño y pilotaje de instrumentos con integrantes de la población transgénero}

No resulta aceptable el uso de instrumentos de medición en personas trans argumentando que su calidad psicométrica ha sido previamente demostrada en otras poblaciones. Las personas trans constituyen un grupo específico cuyas experiencias son profundamente distintas de las de otros colectivos incluidos bajo la denominación general de LGBTI. Instrumentos que funcionan psicométricamente bien en población general o LGBI pueden perfectamente resultar inapropiados para personas transgénero y por ello, se hace necesario adaptarlos y validarlos específicamente en la población destinataria.

Una revisión exhaustiva de los instrumentos con miembros del panel de expertos y/o miembros de la comunidad trans puede permitir, por ejemplo, detectar errores, expresiones confusas 
o poco familiares y emplear el lenguaje sustitutorio apropiado, sobre todo si se trata de instrumentos construidos y utilizados en otro contexto social, cultural y/o lingüístico. Este procedimiento preliminar debe ser seguido, necesariamente, por un pilotaje de estos instrumentos en personas pertenecientes a la comunidad. Este pilotaje, dirigido a detectar eventuales dificultades en la compresión de instrucciones, preguntas y formas de respuesta, pretenden disminuir el riesgo de obtener resultados expuestos a problemas de interpretabilidad y generalización.

En el diseño y evaluación de los instrumentos debe contemplarse también la amplia heterogeneidad interna de la población trans. Por ejemplo, la existencia de identidades de género binarias y no binarias está además atravesada por diversas pertenencias categoriales igualmente relevantes como clase social, origen étnico o racial, orientación sexual, etcétera. Igualmente, resulta importante considerar la intersección del proceso de afirmación de género y las categorías antes señaladas. Por esa razón, el desafío es dar cabida a todos los grupos de modo que queden adecuadamente representados en los instrumentos.

\section{(7) Aplicar instrumentos de modo grupal y realizar debriefing}

La administración colectiva de los instrumentos, aunque con respuesta individual, puede resultar más confortable para los y las participantes de un estudio. Se podría pensar que atender a una sola persona en una entrevista o encuesta cara a cara permitiría más cercanía y controlar mejor la ansiedad del respondiente. La experiencia indica que puede ser una buena estrategia reunir grupos de personas y, antes de administrar los instrumentos, hacer una presentación de los investigadores y de los propios participantes, describir el propósito de la reunión y proporcionar una suerte de debriefing (en el sentido de informar en detalle la investigación, sus objetivos y potencial impacto).

Lo sorprendente fue que las personas trans convocadas a estas reuniones solicitaran ir acompañadas o que, de hecho, llegaran con alguien más que las esperaba mientras ellas respondían. Estas conductas evidenciarían la ansiedad que generaba la situación y la necesidad de sentirse más acompañadas/os (a pesar de que muchas veces fueron contactadas por alguien cercano y conocido de ellas y fueron informadas previamente sobre su participación). La repuesta individual de un cuestionario en una situación grupal, en la que muchas veces coincidían personas conocidas entre sí, resultó ser una excelente forma de contrarrestar la ansiedad, de constituir un espacio de escucha y conversación sumamente informativo que puede ser útil para entender los resultados. Al igual que en la administración de los instrumentos, la aplicación de esta estrategia colectiva en el pilotaje de instrumentos puede orientar de mejor modo las modificaciones que sean necesarias, toda vez que permite generar un vínculo más estrecho con los participantes y acerca la investigación a estos grupos.

El intercambio de experiencias que se produce en estos grupos tan heterogéneos (según edad, nivel socioeconómico, origen étnico, nivel educacional, nacionalidad, etcétera) permite conectarlos con las problemáticas de los otros, propiciando la empatía y la construcción de vínculos sociales en personas que, muchas veces, carecen de redes de apoyo social. Del mismo modo, estos encuentros para responder instrumentos se constituyeron, muchas veces, en espacio de acompañamiento $\mathrm{y}$ soporte emocional en la medida en que los contenidos tratados por los cuestionarios estimulaban la necesidad de compartir ciertas experiencias y emociones, más allá de la simple respuesta a las preguntas. Del mismo modo, esos encuentros constituyen un espacio idóneo para entregar información relativa a sus preocupaciones en diversos ámbitos como salud física y mental, asesoramiento jurídico, ayudas estatales, etcétera). La participación de organizaciones pro-derechos en estas reuniones de administración de instrumentos puede ser también relevante. 


\section{(8) Contemplar modalidades de aplicación asistida}

Aunque se trata de una recomendación de carácter más general, es importante tener en consideración características que siendo propias de toda población, también están presentes en comunidades minoritarias. Por ejemplo, se debe disponer de lugares accesibles para personas en situación de discapacidad y cuestionarios con tipografía aumentada para personas con dificultades visuales. Otra medida es contar con entrevistadores que puedan leer las preguntas y anotar sus respuestas (en el caso que la situación de discapacidad le impida responder por sí mismo). Sabida es la existencia de personas trans que por ser víctimas de rechazo social, han debido abandonar los estudios formales, no siendo capaces de leer y escribir fluidamente. Para ellos/as, el acompañamiento por parte de los administradores de los instrumentos que puedan leer las preguntas y anotar sus respuestas con paciencia y delicadeza de trato resulta sumamente relevante.

Del mismo modo, siempre debe estar presente algún miembro del equipo de investigación que pueda explicar el sentido de las preguntas o dar respuestas a interrogantes que vayan surgiendo. Si bien el espacio de debriefing es el más pertinente para ello, la presencia de personas capaces de responder las consultas a medida que emergen es sumamente importante.

Es habitual que las personas se guarden preguntas para realizarlas una vez terminada la aplicación de sus cuestionarios. Una estrategia interesante es mantener al grupo completo luego de terminada la administración y abrir un espacio para tales preguntas. En general, las preguntas surgirán de la exposición al cuestionario y buscarán obtener información adicional sobre algunos tópicos de interés. Estas conversaciones grupales posteriores pueden ser, si es debidamente informado $\mathrm{y}$ aprobado por el grupo, video o audio-grabados para ser utilizadas como material complementario en el estudio. Esta reunión final permite dar un adecuado cierre a la actividad, responder consultas e intentar integrar experiencias, a la vez que dar contención e informar a los/as participantes en caso de que aparezcan contenidos delicados asociados a su propia experiencia y/o a algunos de los temas indagados por el instrumento.

\section{(9) Utilizar un lenguaje inclusivo y no estigmatizante}

Elegir un lenguaje apropiado no es una cuestión trivial. Resulta inaceptable un lenguaje inoportuno que pueda patologizar las experiencias y elecciones de las personas. Del mismo modo, los juicios morales sobre estas personas no son tolerables en tanto pueden causar daño $\mathrm{u}$ ofender a los/as participantes del estudio (Adams et al., 2017; Bouman et al., 2016). Actualmente, se considera que la mejor forma de asegurar un lenguaje respetuoso es incorporando en la revisión de los materiales, desde el comienzo del estudio, al panel de expertos y miembros de la propia comunidad. Igualmente, mediante esta revisión se debe asegurar la ausencia de expresiones tecnicistas y academicistas.

\section{(10) Convocar a participar en conjunto con agrupaciones $\mathrm{e}$ instituciones patrocinantes}

Quién invita a participar en un estudio es una cuestión muy relevante. Generalmente, aunque los investigadores poseen trayectorias académicas que los vinculan con sus temas de estudio y comunidades de referencia, estas trayectorias, salvo en casos muy específicos, no son necesariamente conocidas por la comunidad. Participar o no de un estudio implica establecer una relación de confianza, asumir que se dará un uso apropiado a la información recolectada y realizar un necesario balance entre los costos y beneficios de implicarse en la investigación. Por definición, las relaciones establecidas suelen ser asimétricas y además, si el invitado a participar desconfía de que 
el estudio pueda genuinamente contribuir a mejorar la calidad de vida de su grupo o a ampliar, en algún sentido, los derechos de las comunidades trans, la decisión a sumarse al estudio puede ser negativa.

Una forma de entregar garantías de un uso apropiado de la información es trabajar muy próximamente con las organizaciones que son reconocidas y están validadas por la comunidad. La articulación con instituciones promotoras de los derechos de las personas trans, con líderes de opinión y personas representativas de su comunidad son la mejor estrategia para ingresar al campo y obtener la participación. En este caso, el trabajo con una variedad de organizaciones de la sociedad civil vinculadas a temáticas relevantes para la comunidad trans resultó fundamental para obtener una cantidad suficiente de respuestas e incluir a un número relativamente amplio de participantes en el presente estudio. Del mismo modo, y en caso de ser posible, puede ser sumamente útil efectuar la aplicación de encuestas o entrevistas en instalaciones de las propias organizaciones, a fin de generar confianza y ofrecer mayor seguridad a las personas participantes.

\section{(11) Atender a la movilidad de la población transgénero}

Es importante considerar que las personas no residen necesariamente en la localidad donde trabajan. Es habitual que en las preguntas sobre este tema, se confundan estos aspectos. Las personas trans constituyen un grupo con gran movilidad geográfica. Una porción importante de ellas trabaja en ciudades diferentes a aquellas donde tiene fijada su residencia debido, en muchos casos, al tipo de labores que han tenido que desempeñar (por ejemplo, comercio sexual) debido al rechazo familiar y social (Casanova y Espinoza, 2018). Este rechazo les ha impedido muchas veces continuar con su formación académica y conseguir mejores trabajos. De allí que, aunque pueda parecer un tema menor, es importante separar, para efectos del análisis posterior, las preguntas referidas al lugar de residencia y al lugar de trabajo. Ello evitará también que se les contacte en ambos lugares y se duplique la convocatoria.

\section{(12) Considerar diferentes trayectorias de transición}

Los cuestionarios deberían incorporar preguntas que contemplen la diversidad de subgrupos y de trayectorias de transición o afirmación de género de las personas trans. La transición es un proceso gradual y muchas veces conflictivo, que puede tomar trayectos muy diversos. No existe una forma única de transitar, como tampoco existe una forma única de vivir subjetivamente ese proceso. Estas diversas posibilidades deben estar debidamente incluidas en las preguntas de un instrumento de investigación. Se pretende justamente visibilizar las diversas trayectorias en lugar de omitirlas o subsumirlas dentro de una forma genérica, logrando así captar esta heterogeneidad y los principales problemas asociados a ella como, por ejemplo, la transfobia internalizada (Austin y Goodman, 2017; Scandurra et al., 2018), el rechazo familiar (Yadegarfard, Meinhold-Bergmann y Ho, 2014), la discriminación social (White, Reisner y Pachankis, 2015), problemas de acceso a atención en salud (Bockting y Keatley, 2011; Brennan, Kuhns, Johnson, Belzer, Wilson y Garofalo, 2012) o a educación (Burns, Leich y Hughes, 2016; Jones, Libson y Hiller, 2006), entre otros.

En definitiva, se trata de construir instrumentos que reconozcan e incorporen la multitud de situaciones vitales problemáticas que entorpecen el proceso de transición, visibilizando dichas experiencias, haciéndolas comunes y no privatizándolas como si fueran asuntos que solo ocurren a una sola persona. Las personas transgénero ya se encuentran familiarizadas con la permanente negación de sus experiencias y los estudios no deberían reproducir aquello. Un adecuado trabajo de diseño de los instrumentos, así como el asesoramiento apropiado pueden servir para evitar omisiones que 
terminen por hacer sentir incómodas/os a las personas participantes.

De igual forma, y como ya hemos apuntado más arriba, muchas personas son parte de diversas comunidades y sus discursos se conforman solapando sus múltiples identidades. También debemos considerar las diversas capas de vulnerabilidad en las que pueden ser situadas socialmente y sus efectos al interpretar los datos.

\section{(13) Escuchar interesadamente y contar con un protocolo de derivación}

Resulta evidente que la negación social de su experiencia vital ha tenido como efecto la invisibilización y estigmatización de las personas trans. Se les ha negado sistemáticamente la posibilidad de expresar sus emociones, creencias, expectativas o modos de ser. Por tales razones, trabajar con ellas implica asumir una posición ética decidida que exige a los investigadores una escucha atenta e interesada. Muchas veces implica explicitar las propias posiciones de los investigadores sobre el estudio (¿Por qué se está haciendo el estudio?) y adherir a una ética del cuidado (Collins, 2000). Esto podría evitar activamente la emisión de microagresiones por parte de los investigadores (Chang y Chung, 2015).

Del mismo modo, es importante mostrar durante la conversación cómo los problemas derivados del proceso de transición tienen su origen en el campo social y cómo la imposición de la norma heterosexual explica buena parte de su posición desaventajada. Lo contrario implicaría privatizar el daño, haciendo aparecer como individuales los efectos adversos que tienen su origen en la estructura social.

El encuentro con otros, cuando se manifiesta un genuino interés por ellos, puede resultar un espacio acogedor y potencialmente reparador, reduciendo expectativas negativas de las personas participantes y permitiéndoles expresarse con confianza sobre temas que son existencialmente relevantes para ellas. Se trata de un encuentro en el cual, muchas veces, el conocimiento surge del reconocimiento del otro. Sin embargo, en este proceso relacional pueden emerger contenidos potencialmente perjudiciales que, hasta ese momento, no eran total o parcialmente advertidos por el participante. Si bien la participación no constituye un encuentro con fines clínicos, es inevitable que muchas veces las personas accedan a vivencias dolorosas de su vida y por ello, debemos tomar precauciones para, dado el caso, proporcionar contención e información o si fuese necesario, derivar a las personas para que reciban ayuda especializada. En este sentido es exigible que se defina previamente la cadena de derivación en caso que emerjan estas situaciones. Aunque en la experiencia que se informa se ha requerido más bien contener y entregar información oportuna, se debe contar con un protocolo que atienda la eventual aparición de casos severamente traumatizados. Lo anterior constituye una exigencia ética ineludible para los investigadores.

\section{(14) Realizar sesiones de interanálisis}

Así como convocar a grupos representativos de la población trans resulta fundamental, efectuar sesiones donde se expongan los principales resultados del estudio y se discutan con integrantes de dicha comunidad puede ser de gran ayuda para comprender su alcance y real significado. Estos encuentros de interanálisis ponen en discusión los hallazgos obtenidos permitiendo el diálogo entre las formas de interpretación propias de la academia con los saberes propios de las personas trans. Si los resultados no tienen sentido para ellas, entonces algo en el diseño, recolección de datos y/o análisis podría estar equivocado.

Del mismo modo, estas sesiones pueden ser importantes para coordinar los diferentes intereses (por ejemplo, generar conocimiento versus defender y promover derechos que se pretenden movilizar en apoyo de una determinada causa) e ir construyendo un lenguaje común que permita el diálogo 
entre la academia, el activismo y la población general. Tenemos la profunda convicción de que el conocimiento debe buscar conexiones prácticas con sus posibles destinatarios interesados.

\section{(15) Contemplar una apropiada difusión de los resultados obtenidos}

Finalmente, un conocimiento que no incide de alguna manera en la política pública o en la agenda social sobre temas de derechos humanos y diversidad sexual, no alcanza plenamente su potencial impacto. Por ello, resulta fundamental la difusión de los resultados por vías más amplias que la sola publicación académica. La mayor parte de esta investigación es financiada con dineros públicos, razón por la cual una devolución apropiada a la sociedad debe ser imperativa en este tipo de estudios. Igualmente, se debe facilitar el acceso a las bases de datos generadas por los proyectos a cualquier persona que fundadamente lo requiera, asegurando de antemano los necesarios resguardos de anonimato y confidencialidad de la información sensible que fueron entregados como garantía a los participantes.

Se debe ofrecer la posibilidad de que cada agrupación que apoyó estos estudios y cada persona que ha participado en ellos reciban un resumen de los principales resultados de los estudios y de ser posible, de las publicaciones académicas o no académicas elaboradas gracias a su apoyo o participación. Esta es otra forma de retribuir por participar, reconociendo, entre otras cosas, la contribución de estas personas. Este reconocimiento permite, además, mantener un vínculo positivo con ellos de cara a futuras experiencias de colaboración.

\section{Discusión}

A esta altura ya parecen incontestables los efectos que el prejuicio y las discriminación tienen sobre el bienestar y la calidad de vida de las personas trans. De allí que investigar con dicha población implique, además de acciones para obtener información relevante y veraz, disponer de los cuidados necesarios para atender a sus necesidades y peculiaridades. Se trata de poblaciones de difícil acceso, estigmatizadas, en las que no existe un marco muestral definido, que poseen indicadores de salud física y psíquica inferiores a los de cualquier otro grupo con el que hayamos trabajado y que, por lo mismo, se requiere extremar las precauciones para no reproducir tipos de relacionamiento que puedan ser dañinos u ofensivos.

Lejos de pretender constituirse en una exigencia para la investigación con población trans, el presente trabajo contiene una serie de sugerencias, provisionales por cierto, que emergen de la reflexión sobre el propio quehacer como investigadores. Simplemente se espera que las guías que aquí se proponen y los razonamientos que las sustentan puedan facilitar el trabajo de investigación con esta población, la cual se encuentra interesada en colaborar en la medida en que se entreguen ciertas garantías de rigor y respeto. Debe reconocerse que esta población está en posesión de un saber al que se debe atender si se quiere arribar a una comprensión cabal de los fenómenos que en ella ocurren.

Además de servir para orientar la planificación y ejecución de estudios, estas recomendaciones pueden resultar también de utilidad como criterios para evaluar la necesaria precaución ética que deben observar los proyectos de investigación con personas trans, aspecto en el cual se ven involucrados los revisores de proyectos, editores y jueces de manuscritos postulados para publicación. Se espera que estas recomendaciones sirvan más como un punto de partida útil para los investigadores que como una lista exhaustiva de consideraciones éticas o prácticas para la investigación con estos colectivos.

En estas recomendaciones, se han omitido deliberadamente algunas cuestiones importantes que son exigencias comunes a toda investigación, cuyo cumplimiento debe ser más riguroso en el caso de grupos vulnerables y que apuntan a los mínimos éticos que 
garanticen que la investigación no subestime los riesgos o el potencial daño de las intervenciones (Santi, 2016). De allí la exigencia de obtener previamente un consentimiento para una participación debidamente informada, en que se respete en todo momento la autonomía de las personas participantes, la que se traduce, por ejemplo, en la omisión de cualquier presión para incorporarse al estudio y en la posibilidad de abandonarlo en cualquier momento sin que eso implique consecuencias de ningún tipo.

Todo investigador debe explicar e informar claramente a los participantes los objetivos del estudio, sus riesgos y efectos previsibles, así como la relevancia que podría tener para quienes participan en él o para sus comunidades, a fin de persuadir respetando la libertad y en ausencia de coerción (Santi, 2016). Del mismo modo, se deben entregar garantías de respeto a la confidencialidad y dado el caso, del anonimato (por ejemplo, explicitando la cadena de custodia de datos). Es por ello que las organizaciones no solo pueden constituirse en aval, sino que también deben operar como supervisores del trabajo en beneficio de su comunidad. En los últimos proyectos realizados, se optó por incorporar la figura de un asesor de datos cuyo rol es colaborar en todo el proceso de producción y análisis de la información; este asesor, además de tener formación investigativa, pertenece a la comunidad con la que se estuvo trabajando. En el caso de la población trans, lo anterior resulta aún más importante dada la situación de vulnerabilidad en que se encuentran en la sociedad. Se trata de grupos estigmatizados, que carecen de recursos materiales, muchas veces en posición de dependencia y en los que se cruzan diversas pertenencias socialmente devaluadas (origen étnico, clase social, sexo/género, etcétera).

Por último, el conocimiento puede ser generado desde una perspectiva denominada activismo basado en la evidencia (Akrich, O’Donnovan y Rabeharisoa, 2013; Rabeharisoa, Moreira y Akrich, 2014). Este enfoque implica articular una variedad de conocimientos, tanto científicamente acreditado como de carácter experiencial, con el fin de explorar situaciones en las que los integrantes del equipo de investigación forman parte de redes que los implican personalmente en sus temas de estudios. Desde esta perspectiva, la investigación sería fruto de un proceso colectivo que reúne a personas que han vivido ciertas experiencias, activistas de derechos y especialistas académicos, con la finalidad de generar conocimiento, sin abstraerse de la inserción en un campo de relaciones políticas y sin negar el deseo de incidir en las políticas públicas y su respectivo monitoreo.

\section{Referencias bibliográficas}

Abdul-Quader, A., Heckathorn, D., Sabin, K. y Saidel, T. (2006). Implementation and analysis of Respondent Driven Sampling: Lesson learned from the field. Journal of Urban Health, 83(7), 1-5.

Adams, N., Pearce, R., Veale, J., Radix, A., Castro, D., Sarkar, A. y Thom, K. C. (2017). Guidance and ethical considerations for undertaking transgender health research and institutional review boards adjudicating this research. Transgender Health, 2(1), 165-175. https://doi.org/10.1089/ trgh.2017.0012.

Akrich, M., O'Donovan, O. y Rabeharisoa, V. (2013). The entanglement of scientific and political claims: toward a new form of patients' activism. Workin Paper 13-MS-07. Paris: Interdisciplinary Institute for Innovation.

American Psychological Association (APA, 2015). Guidelines for Psychological Practice with Transgender and Gender Nonconforming People. American Psychologist, 70(9), 832-864. https://doi.org/10.1037/a0039906

American Psychological Association Task Force on Gender Identity and Gender Variance. (2009). Report of the task force on gender identity and gender variance. Washington, DC: Author.

Aspinall, P. J. y Mitton, L. (2008). Operationalising 'sexual orientation' in routine data collection and equality monitoring in the UK. Culture, Health and Sexuality, 10(1). 57-72. https://doi. org/10.1080/13691050701664460. 
Austin, A. y Goodman, R. (2017). The Impact of Social Connectedness and Internalized Transphobic Stigma on Self-Esteem Among Transgender and Gender Non-Conforming Adults. Journal of Homosexuality, 64(6), 825-841. https://doi.org/10.1080/00918369.2016.12365 87.

Barrientos, J. y Cárdenas, M. (2014). Construction and Validation of a Subjective Scale of Stigma and Discrimination (SISD) for the Gay Men and Transgender Women Population in Chile. Sexuality Research and Social Policy, 11(3), 187-198. https://doi.org/10.1007/s13178-0140150-0

Barrientos, J., Cárdenas, M. y Saiz, J. L. (2016). Hate Crimes in Chile. En E. Dumbar, A. Blanco y D. Crèvecoeur-MacPhail (Eds.), Hate Crimes as Domestic Terrorism, 145-170. LA, California: Praeger Series.

Barrientos, J., Cárdenas, M., Berendsen, P., Vidal, F. y Meneses, A. (2013). Índice compuesto de Estigma y Discriminación hacia hombres homosexuales, otros HSH y mujeres transgénero en Chile (ICED). Santiago: ONUSIDA.

Barrientos, J., Cárdenas, M., Gómez, F. y Guzmán, M. (2014). Gay Men and Male-to-Female Transgender Persons in Chile: An Exploratory Quantitative Study on Stigma, Discrimination, Victimization, Happiness and Social WellBeing. En T. Köllen (Ed), Sexual Orientation and Transgender Issues in Organizations. Global Perspectives on LGBT Workforce Diversity (pp. 253-270). Switzerland: Springer International Publishing. https://doi.org/10.1007/978-3-31929623-4 15.

Barrientos, J., Gómez, F. y Cárdenas, M. (2016). Subjective well-being and levels of clinical symptomatology in a Transwomen sample and Men who have a sex with Men in Chile. Journal of Homosexuality, 63(11), 1502-1516. https:// doi.org/10.1080/00918369.2016.1223346.

Bockting, W. (2014). Transgender identity development. En D. L. Tolman, L. M. Diamond, J. A. Bauermeister, W. H. George, J. G. Pfaus y M. L. Ward (Eds.), APA Handbook of Sexuality and Psychology (pp. 739-758). Washington DC: American Psychological Association.
Bockting, W. y Keatley, J. (2011). Por la salud de las personas Trans. Elementos para el desarrollo de la atención integral de personas Trans y sus comunidades en Latinoamérica y el Caribe. Organización Panamericana de la Salud. https:// pdf.usaid.gov/pdf_docs/PA00JQ81.pdf.

Bockting, W., Knudson, G. y Goldberg, J. M. (2006). Counseling and mental health care for transgender adults and loved ones. International Journal of Transgenderism, 9, 35-82. https:// doi.org/10.1300-J485v09n03_03.

Bouman, W. P., Schwend, A. S., Motmans, J., Smiley, A., Safer, J. D., Deutsch, M. B., Adams, N. J. y Winter, S. (2016). Language and trans health. International Journal of Transgenderism, 18(1), 1-6. https://doi.org/10.1080/15532 739.2016.1262127.

Brennan, J., Kuhns, L. M., Johnson, A. K., Belzer, Wilson, E. C. y Garofalo, R. (2012). Syndemic theory and HIV-related risk among young transgender women: the role of multiple, co-occurring health problems and social marginalization. American Journal of Public Health, 102(9), 1751-1757. https://doi.org/10.2105/ AJPH.2011.300433.

Burns, S., Leitch, R. y Hughes, J. (2016). Barriers and Enablers of Education Equality for Transgender Students. Review of Social Studies, 3(2), 11-20.

Cárdenas, M., Barrientos, J., Gómez, F. y Frías-Navarro, D. (2012). Attitudes toward lesbians and gay men and their relationship with sex role in a sample of Chilean university students. International Journal of Sexual Health, 24, 1-11. Índice WOS. http://doi.org/10.1080/19317611.2012.7 00687

Cárdenas, M. y Yañez, S. (2012). Nuevas formas de muestreo para minorías y poblaciones ocultas: muestras por encuestado conducido en una población de inmigrantes sudamericanos. UniversitasPsychologica, 11(2), 553-560.

Carrara, S., de Garay Hernandez, J., Uziel, A. P., Silva da Conceição, G. M., Panjo, H., de Oliveira Baldanzi, A. C. ... Giami, A. (2019). Body construction and health itineraries: A survey among travestis and trans people in Rio de Janeiro, Brazil. Cadernos de Saúde Pública, 
35(4), 1-14. https://doi.org/10.1590/0102311X00110618.

Casanova, P. y Espinoza, R. (2018). Significados en torno a la atención psicológica durante el proceso de transición de género: una aproximación desde la perspectiva de usuarios/as transgénero en Chile. Al Sur de Todo, 12, 104-116.

Chang, T. K. y Chung, Y. B. (2015). Transgender Microaggressions: Complexity of the Heterogeneity of Transgender Identities. Journal of LGBT Issues in Counseling, 9(3), 217-234. https://doi. org/10.1080/15538605.2015.1068146

Coleman, E., Bockting, W., Botzer, M., Cohen-Kettenis, P., DeCuypere, G., Feldman, J. et al. (2012). Standards of Care for the Health of Transsexual, Transgender, and Gender-Nonconforming People, Version 7. International Journal of Transgenderism, 13(4), 165-232. https://doi.org/10.1080/15532739.2011.700873.

Collins, P. H. (2000). Black feminist thought: Knowledge, consciousness, and the politics of empowerment. New York: Routledge.

Conron, K. J., Scott, G., Stowell, G. S. y Landers, S. J. (2012). Transgender health in Massachusetts: Results from a household probability sample of adults. American Journal of Public Health, 102, 118-122. https://doi.org/10.2105/ AJPH.2011.300315

Emanuel, E. (2005). Undue Inducement: Nonsense on Stilts? The American Journal of Bioethics, 5(5), 9-13. https://doi. org/10.1080/15265160500244959.

Giami, A. y Beaubatie, E. (2014). Gender identification and sex reassignment surgery in the trans population: A survey study in France. Archives of Sexual Behavior, 43(8), 1491-1501. https:// doi.org/10.1007/s10508-014-0382-3.

Grant, J. M., Mottet, L., Tanis, J. E., Harrison, J., Herman, J. y Keisling, M. (2011). Injustice at every turn: A report of the national transgender discrimination survey. Washington, DC: National Center for Transgender Equality and National Gay and Lesbian Task Force.

Hale, J. (2009). Suggested Rules for Non-Transsexuals Writing about Transsexuals, Transsexuality, Transsexualism, or Trans. https://hivdatf. files.wordpress.com/2010/09/suggested-ru- les-for-non-modified.pdf

Harrison, J., Grant, J. y Herman, J. L. (2012). A gender not listed here: Genderqueers, gender rebels, and otherwise in the national transgender discrimination survey. LGBTQ Public Policy Journal at the Harvard Kennedy School, 2(1), 13-24.

Heckathorn, D. (2002). Respondent Driven Sampling II: Deriving population estimates from chain-referral samples of hidden populations. Social Problems, 49(1), 11-34.

Heckathorn, D. (2007). Extensions of Respondent-Driven Sampling: Analyzing continuous variables and controlling for differential recruitment. Sociological Methodology, 37(1), 151-208.

Hereck, G. (2010). Sexual Orientation Differences as Deficits: Science and Stigma in the History of American Psychology. Perspectives on Psychological Science, 5(6), 693-699. https://doi. org/10.1177/1745691610388770.

Jones, D., Libson, M. y Hiller, R. (2006). Sexualidades, Política y Violencia. La Marcha del Orgullo GLTTBI Buenos Aires 2005. Segunda Encuesta. Buenos Aires: Antropofagia.

Krueger, E. A., Divsalar, S., Luhur, W., Choi, S. K. y Meyer, I. H. (2020). TransPop - U.S. Transgender Population Health Survey (Methodology ans Technical Notes). Los Angeles, CA: The Williams Institute.

Keogh, P., Reid, D. y Weatherburn, P. (2006). Lambeth LGBT Matters: The Needs and Experiences of Lesbians, Gay Men, Bisexual and Trans Men and Women in Lambeth. London: Sigma Research.

Luna, F. (2008). Vulnerabilidad: la metáfora de las capas. Jurisprudencia Argentina, IV(1), 60-67.

MacCarthy, S., Reisner, S. L., Nunn, A., PerezBrumer, A. y Operario, D. (2015). The time is now: Attention increases to transgender health in the United States but scientific knowledge gaps remain. LGBT Health, 2(4), 287-291. https://doi.org/10.1089/lgbt.2014.0073

Meier, S. C. y Labuski, C. M. (2013). The demographics of the transgender population. En A. $\mathrm{K}$. Baumle (Ed.), International handbook of the demography of sexuality, 289-327. New York, 
NY: Springer.

Mikalson, P., Pardo, S. y Green, J. (2012). First do no harm: Reducing disparities for lesbian, gay, bisexual, transgender, queer, and questioning populations in California. California: California Reducing Disparities Project.

Miller, J. y Nichols, A. (2012). Identity, sexuality and commercial sex among Sri Lankan nachchi. Sexualities, 15, 554-569. https:/doi. org/10.1177/1363460712446120.

Mitchell, M. y Howarth, C. (2009). Trans Research Review. Manchester: Equality and Human Rights Commission.

Nierman, A. J., Thompson, S. C., Bryan, A. y Mahaffey, A. L. (2007). Gender role beliefs and attitudes towards lesbians and gay men in Chile and the U.S. Sex Roles, 57, 61-67. https://doi. org/10.1007/s11199-007-9197-1.

Rabeharisoa, V., Moreira, T. y Akrich, M. (2014). Evidence-based activism: Patients', users' and activists' groups in knowledge society. BioSocieties, 9(2), 111-128. https://doi.org/10.1057/ biosoc.2014.2.

Salganik, M. (2006). Variance estimation, design effects, and simple size calculation for Respondent-Driven Sampling. Journal of Urban Health, 83(7), 98-112.

Salganik, M. y Heckathorn, D. (2004). Sampling and estimation in hidden populations using Respondent-Driven Sampling. Sociological Methodology, 34, 193-239.

Santi, M. F. (2016). Ética de la investigación en ciencias sociales. Un análisis de la vulnerabilidad en la investigación social. Geneve: Globethics.net Theses 18.

Scandurra, C., Bochicchio, V., Amodeo, A. L., Esposito, C., Valerio, P., Maldonato, N. M., Bacchini, D. y Vitelli, R. (2018). Internalized Transphobia, Resilience, and Mental Health: Applying the Psychological Mediation Framework to Italian Transgender Individuals. International Journal of Environmental Research and Public Health, 15(3), E508. https://doi. org/10.3390/ijerph15030508.

Staples, J., Bird, E., Masters, T. y George, W. (2018). Considerations for culturally sensitive research with transgender adults: A qualitative analysis. Journal of Sex Research, 55(8), 10651076. https://doi.org/10.1080/00224499.2017.1 292419

Tagonist, A. (2009). Fuck you and Fuck your Fucking Thesis: Why I Will Not Participate in Trans Studies. http://tagonist.livejournal. com/199563.html

Vincent, B. W. (2018). Studying trans: recommendations for ethical recruitment and collaboration with transgender participants in academic research. Psychology \& Sexuality, 9(2), 102-116. https://doi.org/10.1080/19419899.201 8.1434558

White, J. M., Reisner, S. L. y Pachankis, J. E. (2015). Transgender stigma and health: A critical review of stigma determinants, mechanisms, and interventions. Social Science \& Medicine, 147, 222-231. https://doi.org/10.1016/j. socscimed.2015.11.010.

Whittle, S., Turner, L. y Al-Alami, M. (2007). Engendered penalties: Transgender and transsexual people's experiences of inequality and discrimination. https://www.ilga-europe.org/ sites/default/files/trans_country_report_engenderedpenalties.pdf

Wolf, E. C. y Dew, B. J. (2012). Understanding risk factors contributing to substance use among MTF transgender persons. Journal of LGBT Issues in Counseling, 6, 237-256. https://doi. org/10.1080/15538605.2012.727743.

Yadegarfard, M., Meinhold-Bergmann, M. E. y Ho, R. (2014).Family rejection, social isolation, and loneliness as predictors of negative health outcomes (depression, suicidal ideation, and sexual risk behavior) among Thai male-to-female transgender adolescents. Journal of LGBT Youth, 11(4), 347-363. https://doi.org/10.1080/1 9361653.2014.910483.

Recibido: 18 de julio de 2019 Aceptado: 3 de noviembre de 2020 